Pacific

Journal of

Mathematics

TOTARO'S QUESTION FOR SIMPLY CONNECTED GROUPS OF LOW RANK

JODI BLACK AND RAMAN PARIMALA

Volume 269 No. 2

June 2014 


\title{
TOTARO'S QUESTION FOR SIMPLY CONNECTED GROUPS OF LOW RANK
}

\author{
JODI BLACK AND RAMAN PARIMALA
}

Let $k$ be a field and let $G$ be a connected linear algebraic group over $k$. In a 2004 paper, Totaro asked whether a torsor $X$ under $G$ and over $k$ which admits a zero cycle of degree $d$ also admits a closed étale point of degree dividing $d$. We consider this question in the setting where $G$ is a simply connected, semisimple group of rank at most 2 and $k$ is of characteristic different from 2.

\section{Introduction}

Serre [1995, p. 233] raised the following question:

Serre's question: Let $k$ be a field and let $G$ be a connected linear algebraic group defined over $k$. Let $X$ be a $G$-torsor over $k$. Suppose $X$ admits a zero cycle of degree 1 . Does $X$ have a $k$-rational point?

An affirmative answer to Serre's question is known in a number of special cases. See, for example, [Sansuc 1981; Bayer-Fluckiger and Lenstra 1990; Black 2011a; 2011b]. Burt Totaro [2004] posed the following generalization of Serre's question:

Totaro's question: Let $k$ be a field and let $G$ be a connected linear algebraic group defined over $k$. Let $X$ be a $G$-torsor over $k$. Suppose $X$ admits a zero cycle of degree $d$. Does $X$ have a closed étale point of degree dividing $d$ ?

An affirmative answer to Totaro's question when $G=\mathrm{PGL}_{n}$ is a classical result in the theory of central simple algebras. Tits [1992] associated to any absolutely simple, linear algebraic $k$-group $G$, an integer $n(G)$. The values of $n(G)$ are shown in Table 1 below, where $v$ denotes the 2 -adic valuation. One can show that for any $G$-torsor $X$, there is a separable field extension $L / k$ such that $X$ has a rational point over $L$ and [ $L: k]$ divides $n(G)^{2}$ [Serre 1995, Section 2.3]. Thus, Tits' construction gives an affirmative answer to Totaro's question provided $n(G)^{2}$ divides $d$. Garibaldi and Hoffmann [2006] give an affirmative answer to Totaro's question for semisimple groups which are of type $G_{2}$, of reduced type $F_{4}$ or simply

Parimala is partially supported by National Science Foundation grant DMS-1001872.

MSC2010: primary 11E72; secondary 11E57, 11E81.

Keywords: zero cycles, Galois cohomology, classical groups, 


\begin{tabular}{c|c}
\hline Type of group & $n(G)$ \\
\hline$A_{n}$ & $2(n+1)$ \\
$B_{n}$ & $2^{n}$ \\
$C_{n}$ & $2^{v(n)+1}$ \\
$D_{n}(n \neq 4)$ & $2^{n+v(n)}$ \\
\hline
\end{tabular}

Table 1. Values of $n(G)$ for classical groups.

connected of type ${ }^{1} E_{6,6}^{0}$ or ${ }^{1} E_{6,2}^{28}$. Their work extended previous results of Totaro [2004] which gave an affirmative answer for split, simply connected groups of type $G_{2}, F_{4}$ and $E_{6}$. Results in [Black 2011b] give an affirmative answer to Totaro's question in the case where $G$ is a simply connected or adjoint, semisimple, classical group and $d$ is prime to $n(G)$.

In this paper we show the following:

Theorem 0.1. The answer to Totaro's question is yes if $k$ is of characteristic different from 2 and $G$ is a semisimple, simply connected, classical group such that rank $G_{\bar{k}} \leq 2$.

\section{Galois cohomology}

Let $k$ be a field, let $k_{s}$ be a separable closure of $k$ and let $\Gamma_{k}=\operatorname{Gal}\left(k_{s} / k\right)$ be the absolute Galois group of $k$. We write $H^{1}(k, G)$ for the first Galois cohomology set $H^{1}\left(\Gamma_{k}, G\left(k_{s}\right)\right)$. Given any finite field extension $L / k$ there is a canonical restriction map $H^{1}(k, G) \rightarrow H^{1}(L, G)$. If $\lambda \in H^{1}(k, G)$ is any element, we write $\lambda_{L}$ for the image of $\lambda$ under the restriction map $H^{1}(k, G) \rightarrow H^{1}(L, G)$.

For our convenience, we will consider the formulation of Totaro's question in Galois cohomology:

Totaro's question: Let $k$ be a field and let $G$ be a connected linear algebraic group defined over $k$. Let $\left\{L_{i}\right\}_{1 \leq i \leq m}$ be a set of finite field extensions of $k$ and let $d=\operatorname{gcd}\left\{\left[L_{i}: k\right]_{1 \leq i \leq m}\right\}$. If $\lambda_{L_{i}}=1$ for all $i$, is there a finite, separable field extension $F$ of $k$ such that $\lambda_{F}=1$ and $[F: k]$ divides $d$ ?

\section{Results}

In this section, we consider Totaro's question for various groups $G$.

The case $G=\mathrm{SL}_{1}(A)$.

Theorem 2.1. The answer to Totaro's question is yes if $G=\mathrm{SL}_{1}(A)$ for A a central simple algebra over $k$ of prime index.

Proof. Let $\left\{L_{i}\right\}_{1 \leq i \leq m}$ be a set of finite field extensions of $k$ and suppose $\lambda \in$ $H^{1}\left(k, \mathrm{SL}_{1}(A)\right)$ is an element such that $\lambda_{L_{i}}=1$ for all $i$. Let $d=\operatorname{gcd}\left\{\left[L_{i}: k\right]_{1 \leq i \leq m}\right\}$. 
We will find $F / k$ separable such that $\lambda_{F}=1$ and $[F: k]$ divides $d$.

Since by [Knus et al. 1998, Theorem 29.2], $H^{1}\left(k, \mathrm{GL}_{1}(A)\right)=1$, the short exact sequence

$$
1 \longrightarrow \mathrm{SL}_{1}(A) \longrightarrow \mathrm{GL}_{1}(A) \stackrel{\mathrm{Nrd}}{\longrightarrow} G_{m} \longrightarrow 1
$$

induces the long exact sequence

$$
A^{*} \stackrel{\mathrm{Nrd}}{\longrightarrow} k^{*} \longrightarrow H^{1}\left(k, \mathrm{SL}_{1}(A)\right) \longrightarrow 1
$$

in Galois cohomology, where Nrd is the reduced norm. By (2.1.1) above,

$$
H^{1}\left(k, \mathrm{SL}_{1}(A)\right) \cong k^{*} / \operatorname{Nrd}\left(A^{*}\right),
$$

and we can identify $\lambda$ with the class of an element of $k^{*}$ which is $\operatorname{in} \operatorname{Nrd}\left(A_{L_{i}}\right)$ for all $i$. For simplicity, we will also refer to this element as $\lambda$. Let the index of $A$ be $s$ and choose $L$ contained in $A$ a separable field extension of $k$ of degree $s$ which splits $A$ [Gille and Szamuely 2006, Propositions 4.5.3 and 4.5.4]. Then $\operatorname{Nrd}\left(A_{L}\right)=L^{*}$ and $\lambda$ is in $\operatorname{Nrd}\left(A_{L}\right)$. So if $s$ divides $d$ we may take $F=L$. Recall that $s$ is prime. So if $s$ does not divide $d$ then $\operatorname{gcd}(s, d)=1$. It is well known that $N_{L / k}\left(\operatorname{Nrd}\left(A_{L}\right)\right) \subseteq \operatorname{Nrd}(A)$. In particular, $\lambda^{s}=N_{L / k}(\lambda)$ is in $\operatorname{Nrd}(A)$. Since $\operatorname{Nrd}(A)$ is a group and $N_{L_{i} / k}(\lambda) \in \operatorname{Nrd}(A)$ for all $i$, we find that $\lambda^{d}$ is $\operatorname{in} \operatorname{Nrd}(A)$. In turn, $\lambda$ is in $\operatorname{Nrd}(A)$ and we can take $F=k$.

\section{The case $G=\mathrm{SU}(A, \sigma)$.}

Theorem 2.2. The answer to Totaro's question is yes if $k$ is of characteristic different from 2 and $G=\mathrm{SU}(A, \sigma)$ for a central simple algebra $A$ of degree 3 over $K$, $k=K^{\sigma}$ and $[K: k]=2$.

Proof. Let $\left\{L_{i}\right\}_{1 \leq i \leq m}$ be a set of finite field extensions of $k$ and suppose $\lambda \epsilon$ $H^{1}(k, \operatorname{SU}(A, \sigma))$ is an element such that $\lambda_{L_{i}}=1$ for all $i$. Let $d=\operatorname{gcd}\left\{\left[L_{i}: k\right]\right\}$. We will find $F / k$ separable such that $\lambda_{F}=1$ and $[F: k]$ divides $d$.

The case where $d$ is coprime to 2 and 3 was covered in [Black 2011b, Theorem 3.4]. If $6 \mid d$, we take $L$ to be a separable extension of $K$ of degree dividing 3 which splits $A$. Since $K / k$ is Galois, $L / k$ is separable of degree dividing 6. Since $H^{1}(K, \operatorname{SU}(A, \sigma))=H^{1}\left(K, \mathrm{SL}_{1}(A)\right)$ and $L$ splits $A, H^{1}(L, \operatorname{SU}(A, \sigma))=\{1\}$ by Hilbert's Theorem 90. Therefore, for any $\lambda \in H^{1}(k, \operatorname{SU}(A, \sigma)), \lambda_{L}=1$ and we can take $F=L$. Now suppose $2 \mid d$ and $3 \nmid d$. Fix an index $i$ such that $\left[L_{i}: k\right]$ is prime to 3 and $\lambda_{L_{i}}=1$. Consider $L_{i} K$, the compositum of $L_{i}$ and $K$. Since, by assumption, 3 is prime to $\left[L_{i}: k\right]$, and $[K: k]=2$, we know that 3 is prime to $\left[L_{i} K: k\right]$. Therefore, 3 is prime to $\left[L_{i} K: K\right]$. Let $L$ be a separable splitting field of $A$ such that $[L: K]$ is equal to the index of $A$. Since $\operatorname{deg}_{K}(A)=3$, either $[L: K]=1$ or $[L: K]=3$. In either case, $L, L_{i} K$ is a pair of field extensions of $K$ such that $\lambda_{L}=1=\lambda_{L_{i} K}$ and 
$\operatorname{gcd}\left\{[L: K],\left[L_{i} K: K\right]\right\}$ is 1 . Since $H^{1}(K, \operatorname{SU}(A, \sigma))=H^{1}\left(K, \mathrm{SL}_{1}(A)\right)$ we have $\lambda_{K}=1$ by Theorem 2.1, and we can take $F=K$. The final setting to consider is the case where $3 \mid d$ and $2 \nmid d$. Since $d$ is odd, we can fix an index $i$ such that $\left[L_{i}: k\right]$ is odd and $\lambda_{L_{i}}=1$. Let $R_{K / k} G_{m}$ be the Weil transfer of $G_{m}$ and let $R_{K / k}^{1} G_{m}$ be defined as the kernel of the norm map $N_{K / k}: R_{K / k} G_{m} \rightarrow G_{m}$. The short exact sequence

$$
1 \rightarrow \mathrm{SU}(A, \sigma) \rightarrow U(A, \sigma) \rightarrow R_{K / k}^{1} G_{m} \rightarrow 1
$$

induces the commutative diagram

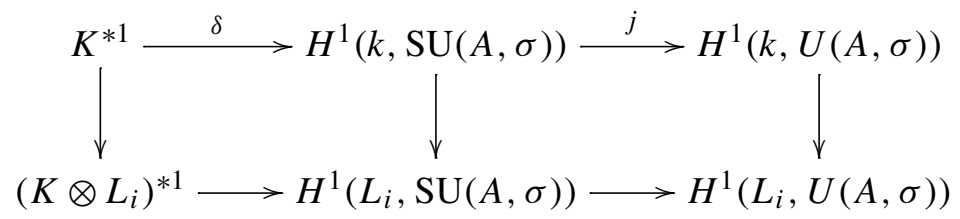

where $K^{* 1}$ and $\left(K \otimes L_{i}\right)^{* 1}$ denote the norm-one elements in $K^{*}$ and $\left(K \otimes L_{i}\right)^{*}$ respectively. By a result of Bayer-Fluckiger and Lenstra [1990, Theorem 2.1], $j(\lambda)=1$. In particular, we can choose $\alpha \in K^{* 1}$ such that $\delta(\alpha)=\lambda$. In the case where $A$ is split, $H^{1}(K, \mathrm{SU}(A, \sigma))=H^{1}\left(K, \mathrm{SL}_{1}(A)\right)=\{1\}$. Then, since $K$ and $L_{i}$ are field extensions of coprime degree with $\lambda_{K}=\lambda_{L_{i}}=1$, the desired result holds by [Black 2011b, Theorem 4.4]. Since $\operatorname{deg}(A)=3$, if $A$ is not split, then $A$ is a division algebra and by [Albert 1963] (see also [Knus et al. 1998, Theorem 19.14]), there is a $k$-subalgebra $L$ of $A$ such that $L / k$ is étale of degree three. Since $A$ is division, $L$ is a field. Consider the diagram

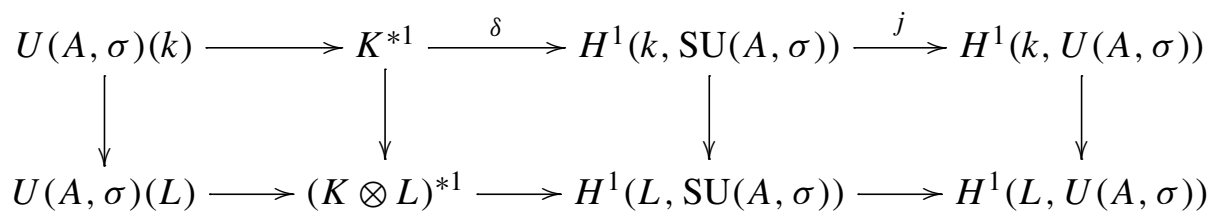

For $x \in(K \otimes L)^{* 1}$, write $x=y^{-1} \bar{y}$ for $y \in(K \otimes L)^{*}$ where ${ }^{-}$denotes the nontrivial automorphism of $K / k$. Since $A \otimes L$ is split, $y$ is a reduced norm from $A \otimes L$. In view of [Merkurjev 1995, Proposition 6.1], the image of $\operatorname{Nrd}\left(U(A, \sigma) \rightarrow(K \otimes L)^{* 1}\right)$ contains $x$. Thus $\lambda_{L}=1$ and we may take $F=L$.

The case $G=\operatorname{Spin}(q)$. The following result will be useful:

Proposition 2.3. Let $k$ be a field of characteristic different from 2 and let $q$ be a quadratic form over $k$ of dimension $\leq 5$. Let $\lambda \in H^{1}(k, \operatorname{Spin}(q))$ be any element. Then there exists a (separable) field extension $F$ of $k$ such that $[F: k]$ divides 2 and $\lambda_{F}=1$. 
Proof. Consider the short exact sequence

$$
1 \longrightarrow \mu_{2} \stackrel{i}{\longrightarrow} \operatorname{Spin}(q) \stackrel{\pi}{\longrightarrow} O^{+}(q) \longrightarrow 1,
$$

which induces the exact sequence in Galois cohomology

$$
H^{1}\left(k, \mu_{2}\right) \stackrel{i}{\longrightarrow} H^{1}(k, \operatorname{Spin}(q)) \stackrel{\pi}{\longrightarrow} H^{1}\left(k, O^{+}(q)\right) .
$$

The pointed set $H^{1}\left(k, O^{+}(q)\right)$ classifies quadratic forms over $k$ of the same dimension and discriminant as $q$. Let $q^{\prime}=\pi(\lambda)$. Then $q \perp-q^{\prime}$ has even dimension, trivial discriminant and trivial Clifford invariant since $q^{\prime}$ is in the image of $\pi$. Thus $q \perp-q^{\prime} \in I^{3}(k)$.

First consider the case where $\operatorname{dim}(q)<4$. Then, $\operatorname{dim}\left(q \perp-q^{\prime}\right)<8$ and by the Arason-Pfister Hauptsatz [Lam 1980, Chapter X, Hauptsatz 5.1], $q \perp-q^{\prime}$ is hyperbolic. Equivalently, $q \cong q^{\prime}$ and $q^{\prime}=1$ in $H^{1}\left(k, O^{+}(q)\right)$. Using the exactness of (2.3.1), choose $\eta$ in $H^{1}\left(k, \mu_{2}\right)$ such that $i(\eta)=\lambda$. Since $H^{1}\left(k, \mu_{2}\right) \cong k^{*} / k^{* 2}$ we can choose $F / k$ a field extension of degree at most 2 such that $\eta_{F}=1 \in H^{1}\left(F, \mu_{2}\right)$. By commutativity of (2.3.2) below, $\lambda_{F}=1$ in $H^{1}(F, \operatorname{Spin}(q))$.

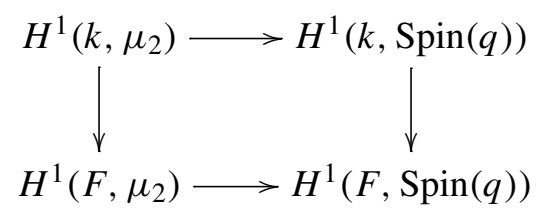

Suppose instead that $\operatorname{dim}(q)=4$. Let $d=\operatorname{disc}(q)$ and write $q=a\langle 1, b, c, b c d\rangle$. By [Lam 1980, Chapter XII, Proposition 2.4], there is an element $\alpha \in k^{*}$ such that $q^{\prime} \cong \alpha q$ and we may write $q \perp-q^{\prime} \cong\langle 1,-\alpha\rangle q=a\langle 1,-\alpha\rangle\langle 1, b, c, b c d\rangle$. Let $e_{2}$ be the map from $I^{2}(k) \rightarrow H^{2}\left(k, \mu_{2}\right)$ induced by the Clifford invariant. Since $q \perp-q^{\prime} \in I^{3}(k), e_{2}\left(q \perp-q^{\prime}\right)=(d) \cup(\alpha)=0 \in H^{2}\left(k, \mu_{2}\right)$ [Elman et al. 2008, 16.2] and so $\langle 1,-\alpha,-d, \alpha d\rangle$ is hyperbolic. Equivalently, $\langle 1,-\alpha\rangle d \cong\langle 1,-\alpha\rangle$ and $q \perp-q^{\prime} \cong a\langle 1,-\alpha\rangle\langle 1, b, c, b c\rangle=a\langle 1,-\alpha\rangle\langle 1, b\rangle\langle 1, c\rangle$. Let $F=k(\sqrt{-b})$. Then $[F: k] \leq 2,\left(q \perp-q^{\prime}\right)_{F}$ is hyperbolic and $q_{F}^{\prime}=1 \in H^{1}\left(F, O^{+}(q)\right)$. Consider the diagram

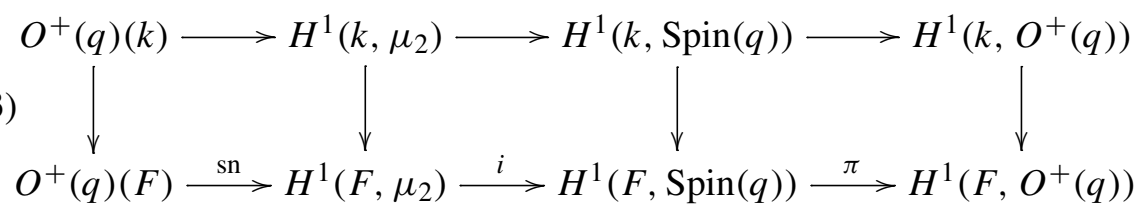

By commutativity of the right rectangle, $\pi\left(\lambda_{F}\right)=1$ and by the exactness of the bottom row, $\lambda_{F} \in \operatorname{im}(i)$. But since $q \cong a\langle 1, b, c, b c d\rangle, q_{F}$ is isotropic. Thus, the 
spinor norm sn : $O^{+}(q)(F) \rightarrow H^{1}\left(F, \mu_{2}\right)$ is onto [Baeza 1978, p. 78] and therefore, since $\lambda_{F} \in \operatorname{im}(i), \lambda_{F}=1$.

Now suppose $\operatorname{dim}(q)=5$. Since $q \perp-q^{\prime}$ is a rank 10 form in $I^{3}(k)$, it is isotropic [Lam 1980, Chapter XII, Proposition 2.8]. Therefore $q$ and $q^{\prime}$ have a common slot and we can write $q=\langle a\rangle \perp q_{1}$ and $q^{\prime}=\langle a\rangle \perp q_{2}$. Since $q_{1} \perp-q_{2} \in I^{3} k$ is rank 8 , we can proceed as in the rank 4 case and find a field extension $F$ of $k$ of degree at most 2 such that $\left(q_{1} \perp-q_{2}\right)_{F}$ is hyperbolic and $\left(q_{1}\right)_{F}$ is isotropic. By the ArasonPfister Hauptsatz, $\left(q \perp-q^{\prime}\right)_{F}$ is hyperbolic and thus $q_{F} \cong q_{F}^{\prime}$ and $\pi\left(\lambda_{F}\right)=q_{F}^{\prime}=$ $1 \in H^{1}\left(F, O^{+}(q)\right)$. Thus $\lambda_{F}$ is in the image of $i: H^{1}\left(F, \mu_{2}\right) \rightarrow H^{1}(F, \operatorname{Spin}(q))$. However, $\left(q_{1}\right)_{F}$ being isotropic, $q_{F}$ is isotropic and sn : $O^{+}(q)(F) \rightarrow H^{1}\left(F, \mu_{2}\right)$ is onto. Therefore, $i$ is the zero map and $\lambda_{F}=1$.

Theorem 2.4. The answer to Totaro's question is yes if $k$ is of characteristic different from 2 and $G=\operatorname{Spin}(q)$ for $q$ a quadratic form of dimension $\leq 5$.

Proof. Let $\left\{L_{i}\right\}_{1 \leq i \leq m}$ be a set of finite field extensions of $k$ and suppose $\lambda \in$ $H^{1}(k, \operatorname{Spin}(q))$ is an element such that $\lambda_{L_{i}}=1$ for all $i$. Let $d=\operatorname{gcd}\left\{\left[L_{i}: k\right]\right\}$. We want to find $F / k$ separable such that $\lambda_{F}=1$ and $[F: k]$ divides $d$. If $d$ is odd we are done by [Black 2011b, Theorem 3.7] and can take $F=k$. If $d$ is even, by Proposition 2.3, there is a separable extension $F / k$ of degree at most 2 such that $\lambda_{F}=1$.

Theorem 2.5. The answer to Totaro's question is yes if $k$ is of characteristic different from 2 and $G=\operatorname{Sp}(A, \sigma)$ where $A$ is a central simple algebra with symplectic involution and $\operatorname{deg}(A)$ is 2 or 4 .

Proof. Let $q$ be a quadratic form of dimension 3 (resp. 5) with trivial discriminant. Then the even Clifford algebra $A=C_{0}(V, q)$ is a central simple algebra of degree 2 (resp. 4) and the canonical involution on the Clifford algebra is symplectic and $\operatorname{Spin}(q) \cong \operatorname{Sp}(A, \sigma)$ [Knus et al. 1998, Section 15.C]. Moreover, every algebra $A$ of degree 2 or 4 with a symplectic involution arises in this way. Thus, a positive answer to Totaro's question for $\operatorname{Sp}(A, \sigma)$ follows from Proposition 2.3.

\section{The case $G=\operatorname{Spin}(A, \sigma)$.}

Theorem 2.6. The answer to Totaro's question is yes if $k$ is of characteristic different from 2 and $G=\operatorname{Spin}(A, \sigma)$, where $A$ is a central simple algebra of degree 4 over $k$ and $\sigma$ is an orthogonal involution on $A$.

Proof. Let $\left\{L_{i}\right\}_{1 \leq i \leq m}$ be a set of finite field extensions of $k$ and suppose $\lambda \in$ $H^{1}(k, \operatorname{Spin}(A, \sigma))$ is an element such that $\lambda_{L_{i}}=1$ for all $i$. Let $d=\operatorname{gcd}\left\{\left[L_{i}: k\right]\right\}$. We will find $F / k$ separable such that $\lambda_{F}=1$ and $[F: k]$ divides $d$.

By [Black 2011b, Theorem 3.7], when $d$ is odd we may take $F=k$. So we may suppose that $d$ is even. Suppose $(A, \sigma)$ has trivial discriminant. Then 
$(A, \sigma) \cong\left(Q_{1} \otimes Q_{2}, \tau_{1} \otimes \tau_{2}\right)$ [Knus et al. 1998, Corollary 15.12], where $Q_{1}$ and $Q_{2}$ are quaternion algebras with the symplectic involution given by conjugation. In turn $\operatorname{Spin}(A, \sigma) \cong \mathrm{SL}_{1}\left(Q_{1}\right) \times \mathrm{SL}_{1}\left(Q_{2}\right)$ [Knus et al. 1998, Corollary 15.13]. There exist $\lambda_{1}, \lambda_{2} \in k^{*}$ such that $\lambda=\left(\bar{\lambda}_{1}, \bar{\lambda}_{2}\right)$ with $\bar{\lambda}_{i} \in k^{*} / \operatorname{Nrd}\left(Q_{i}\right) \cong H^{1}\left(k, \operatorname{SL}_{1}\left(Q_{i}\right)\right)$ for $i=1,2$. In the case $4 \mid d$, let $F_{1}, F_{2}$ be extensions of $k$ of degree at most 2 which split $Q_{1}$ and $Q_{2}$ respectively. Then $\lambda_{F_{1} F_{2}}=1$ and $\left[F_{1} F_{2}: k\right]$ divides 4 . In the case $2 \mid d$ and $4 \nmid d$, we can fix an $L_{j} / k$ such that $\left[L_{j}: k\right]=2 m$, where $m$ is odd and $\lambda_{L_{j}}=1$. Following arguments as in [Garibaldi and Hoffmann 2006, Lemma 1.5] we suppose without loss of generality that $k \subseteq L \subseteq L_{j}$ with $[L: k]$ odd, $\left[L_{j}: L\right]=2$ and $\lambda_{L_{j}}=1$. Let $N_{Q_{1}}, N_{Q_{2}}$ be the norm forms for the quaternion algebras $Q_{1}, Q_{2}$ respectively and let $\phi_{1}=\left\langle 1,-\lambda_{1}\right\rangle N_{Q_{1}}$ and $\phi_{2}=\left\langle 1,-\lambda_{2}\right\rangle N_{Q_{2}}$. The fact that $\lambda_{L_{j}}=1$ implies that $\phi_{1}, \phi_{2}$ are hyperbolic over $L_{j}$. Then by [Garibaldi and Hoffmann 2006, Lemma 1.4] there exists $\mu \in k^{*}$ such that $\phi_{1} \cong\langle 1, \mu\rangle \tilde{\phi}_{1}$ and $\phi_{2} \cong\langle 1, \mu\rangle \tilde{\phi}_{2}$, where $\tilde{\phi}_{1}, \tilde{\phi}_{2}$ are 2-fold Pfister forms. Let $F=k(\sqrt{-\mu})$. Then $\phi_{1}, \phi_{2}$ are hyperbolic over $F$ and thus $\lambda_{1} \in \operatorname{Nrd}\left(Q_{1_{F}}\right)$ and $\lambda_{2} \in \operatorname{Nrd}\left(Q_{2_{F}}\right)$. That is, $\lambda_{F}=1$. Also, $F / k$ is separable and degree at most 2 by construction.

Suppose instead that $(A, \sigma)$ has nontrivial discriminant. One can associate to $(A, \sigma)$ its Clifford algebra $Q$, which is a quaternion algebra with center $K=k(\sqrt{\delta})$, where $\delta=\operatorname{disc}(A, \sigma)$ [Knus et al. 1998, Theorem 15.7]. Then $\operatorname{Spin}(A, \sigma)=$ $R_{K / k} \mathrm{SL}_{1}(Q)$ [Knus et al. 1998, Proposition 15.10] and $H^{1}(k, \operatorname{Spin}(A, \sigma))=$ $H^{1}\left(K, \mathrm{SL}_{1}(Q)\right)$. If $Q$ is split, $\lambda=1$ and we take $F=k$. So suppose $Q$ is not split. If $4 \mid d$ we can take $F$ a splitting field of $Q$ such that $F / K$ is a separable extension of degree 2 . Since

$$
H^{1}(F, \operatorname{Spin}(A, \sigma))=H^{1}\left(K \otimes F, \mathrm{SL}_{1}(Q)\right) \cong H^{1}\left(F \times F, \mathrm{SL}_{1}(Q)\right)=\{1\},
$$

we obtain $\lambda_{F}=1$. Further $[F: k]=4$, and since $F / K$ and $K / k$ are separable, $F / k$ is separable. We are left to consider the case where $(A, \sigma)$ has nontrivial discriminant and $4 \nmid d$ and $2 \mid d$.

Consider the short exact sequence

$$
1 \rightarrow R_{K / k} \mathrm{SL}_{1}(Q) \rightarrow R_{K / k} \mathrm{GL}_{1}(Q) \rightarrow R_{K / k} G_{m} \rightarrow 1
$$

which induces

$$
\mathrm{GL}_{1}(Q)(K) \stackrel{\mathrm{Nrd}}{\longrightarrow} K^{*} \longrightarrow H^{1}\left(K, \mathrm{SL}_{1}(Q)\right) \longrightarrow 1
$$

Choose $\lambda \in H^{1}\left(K, \mathrm{SL}_{1}(Q)\right)$ such that $\lambda_{L_{i}}=1$ for all $i$ and let $\beta \in K^{*}$ satisfy $\delta(\beta)=\lambda$. Following [Garibaldi and Hoffmann 2006, Lemma 1.5], we may suppose that $\lambda_{L_{j}}=1$ where $k \subseteq L \subseteq L_{j}$ and $\left[L_{j}: L\right]=2$. 


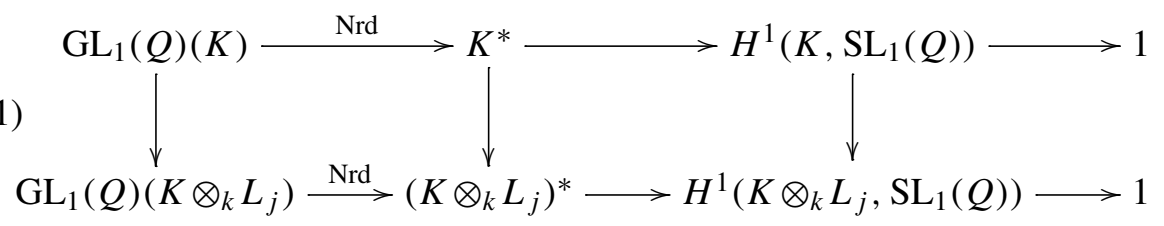

Write $L_{j}=L(\sqrt{a})$ for $a \in L^{*} / L^{* 2}$. Let $f$ be the norm form on $Q$ and let $f^{0}$ denote the norm form restricted to the traceless elements of $Q$, which we denote by $Q^{0}$. Since $\lambda_{L_{j}}=0$, choose $x_{0}, y_{0} \in Q \otimes L$ such that

$$
\beta=f\left(x_{0}+y_{0} \sqrt{a}\right) .
$$

If $y_{0}=0$ we have $\beta \in \operatorname{Nrd}(Q \otimes L)$, and, $L / K$ being of odd degree, this implies $\beta \in \operatorname{Nrd}(Q)$. We take $F=k$. So suppose $y_{0} \neq 0$. Since $Q$ is a division algebra, $f\left(y_{0}\right) \neq 0$ and

$$
\beta=f\left(x_{0}\right)+a f\left(y_{0}\right) .
$$

If we let $b_{f}$ denote the adjoint bilinear form, we have

$$
b_{f}\left(x_{0}, y_{0}\right)=0
$$

and

$$
\beta f\left(y_{0}^{-1}\right)=f\left(x_{0} y_{0}^{-1}\right)+a,
$$

where the reduced trace $\operatorname{trd}\left(x_{0} y_{0}^{-1}\right)$ vanishes by (2.6.4). Therefore,

$$
\beta f\left(y_{0}^{-1}\right)=f^{0}\left(x_{0} y_{0}^{-1}\right)+a .
$$

Let $f=f_{1}+\sqrt{\delta} f_{2}$ with $f_{1}$ and $f_{2}$ quadratic forms on $Q$ with values in $k$. Further let $f^{0}=f_{1}^{0}+\sqrt{\delta} f_{2}^{0}$ where $f_{1}^{0}, f_{2}^{0}$ are quadratic forms on $Q^{0}$ with values in $k$. Setting $z_{0}=y_{0}^{-1}$ and $w_{0}=x_{0} y_{0}^{-1}$, we have

$$
\begin{aligned}
& a=\beta_{1} f_{1}\left(z_{0}\right)+\beta_{2} \delta f_{2}\left(z_{0}\right)-f_{1}^{0}\left(w_{0}\right), \\
& 0=\beta_{1} f_{2}\left(z_{0}\right)+\beta_{2} f_{1}\left(z_{0}\right)-f_{2}^{0}\left(w_{0}\right),
\end{aligned}
$$

with $z_{0} \in Q \otimes L$ and $w_{0} \in Q^{0} \otimes L$. Define $k$-quadratic forms $q_{1}: Q \oplus Q^{0} \rightarrow k$ and $q_{2}: Q \oplus Q^{0} \rightarrow k$ by

$$
\begin{aligned}
& q_{1}(z, w)=\beta_{1} f_{1}(z)+\beta_{2} \delta f_{2}(z)-f_{1}^{0}(w), \\
& q_{2}(z, w)=\beta_{1} f_{2}(z)+\beta_{2} f_{1}(z)-f_{2}^{0}(w),
\end{aligned}
$$

for $z \in Q$ and $w \in Q_{0}$. Since $y_{0} \neq 0, z_{0}=y_{0}^{-1} \neq 0$ and $\left(z_{0}, w_{0}\right)$ is a nontrivial zero of $q_{2}$ over $L$. Then by Springer's theorem [1952], $q_{2}$ has a nontrivial zero $\left(z_{1}, w_{1}\right)$ 
over $k$. By a general position argument, we may assume that $z_{1} \neq 0$. Let

$$
\alpha=\beta_{1} f_{1}\left(z_{1}\right)+\beta_{2} \delta f_{2}\left(z_{1}\right)-f_{1}^{0}\left(w_{1}\right) .
$$

We have

$$
0=\beta_{1} f_{2}\left(z_{0}\right)+\beta_{2} f_{1}\left(z_{0}\right)-f_{2}^{0}\left(w_{1}\right) .
$$

Adding these two equations, we find

$$
\alpha=\beta f\left(z_{1}\right)-f^{0}\left(w_{1}\right),
$$

or, equivalently,

$$
\beta f\left(z_{1}\right)=\alpha+f^{0}\left(w_{1}\right) .
$$

Let $F=k(\sqrt{\alpha})$. Then $[F: k] \leq 2,\left(\sqrt{\alpha}+w_{1}\right) z_{1}^{-1} \in Q_{F}$ and $\beta=\operatorname{Nrd}\left(\left(\sqrt{\alpha}+w_{1}\right) z_{1}^{-1}\right)$. Thus, $\lambda_{F}=1$.

Theorem 2.7. The answer to Totaro's question is yes if $k$ is of characteristic different from 2 and $G=\mathrm{SU}(A, \sigma)$ where $A$ is a quaternion algebra with unitary involution $\sigma$.

Proof. The norm algebra $N_{K / k}(A, \sigma)$ equals $(B, \tau)$ for $B$ a central simple algebra of degree 4 and $\tau$ an orthogonal involution on $B$. Since $\operatorname{Spin}(B, \tau) \cong \operatorname{SU}(A, \sigma)$, that Totaro's question has an affirmative answer in this case is a consequence of Theorem 2.6.

\section{Conclusion}

Theorem 3.1. The answer to Totaro's question is yes for $k$ a field of characteristic different from 2 and $G$ a simply connected, semisimple, classical group of rank $\leq 2$.

Proof. We suppose in all cases that $G$ is simply connected and semisimple and that the rank of $G_{\vec{k}} \leq 2$. If $G$ is of type ${ }^{1} A_{1}$ or ${ }^{1} A_{2}$ then $G$ is of the form $\operatorname{SL}_{1}(A)$ for $A$ a central simple algebra of degree 2 or 3 [Knus et al. 1998, Theorem 26.9]. A positive answer to Totaro's question for a group of this form was shown in Theorem 2.1. If $G$ is of type ${ }^{2} A_{1}$ then $G=\operatorname{SU}(A, \sigma)$ for $A$ a central simple algebra of degree 2 with unitary involution $\sigma$. The proof for this case was given in Theorem 2.7. If $G$ is of type ${ }^{2} A_{2}$ then $G$ is of the form $\operatorname{SU}(A, \sigma)$, where $A$ is a central simple algebra of degree 3 with unitary involution $\sigma$ [Knus et al. 1998, Theorem 26.9]. Thus an affirmative answer to Totaro's question for a group of type ${ }^{2} A_{2}$ follows from Theorem 2.2 above. If $G$ is of type $B_{1}$ or $B_{2}$, then $G=\operatorname{Spin}(q)$ for $q$ a quadratic form of dimension 3 or 5 [Knus et al. 1998, Theorem 26.12] and the desired result was proven in Theorem 2.4. If $G$ is of type $C_{1}$ or $C_{2}$, then $G=\operatorname{Sp}(A, \sigma)$, where $A$ is a central simple algebra of degree 2 or 4 and $\sigma$ is a symplectic involution 
on $A$. The proof of our result in this case was covered in Theorem 2.5. If $G$ is of type $D_{2}$ then either $G=\operatorname{Spin}(q)$ for $q$ a quadratic form of dimension 2 or 4 or $G$ is of the form $\operatorname{Spin}(A, \sigma)$ for $A$ a central simple algebra over $k$ of degree 4 and $\sigma$ an orthogonal involution on $A$ [Knus et al. 1998, Theorem 26.15]. In the first case the desired results follows from Theorem 2.4 and in the latter it follows from Theorem 2.6.

Remark 3.2. Since Garibaldi and Hoffman [2006] have given a proof in the case $G$ is of type $G_{2}$, Totaro's question has a positive answer for any simply connected, semisimple group of rank $\leq 2$.

\section{References}

[Albert 1963] A. A. Albert, "On involutorial associative division algebras", Scripta Math. 26 (1963), 309-316. MR 31 \#3451 Zbl 0147.28702

[Baeza 1978] R. Baeza, Quadratic forms over semilocal rings, Lecture Notes in Mathematics 655, Springer, Berlin, 1978. MR 58 \#10972 Zbl 0382.10014

[Bayer-Fluckiger and Lenstra 1990] E. Bayer-Fluckiger and H. W. Lenstra, Jr., "Forms in odd degree extensions and self-dual normal bases", Amer. J. Math. 112:3 (1990), 359-373. MR 91h:11030 Zbl 0729.12006

[Black 2011a] J. Black, "Implications of the Hasse principle for zero cycles of degree one on principal homogeneous spaces", Proc. Amer. Math. Soc. 139:12 (2011), 4163-4171. MR 2012g:11071 Zbl 1257.11038

[Black 2011b] J. Black, "Zero cycles of degree one on principal homogeneous spaces", J. Algebra 334 (2011), 232-246. MR 2012g:12008 Zbl 05990153

[Elman et al. 2008] R. Elman, N. Karpenko, and A. S. Merkurjev, The algebraic and geometric theory of quadratic forms, American Mathematical Society Colloquium Publications 56, American Mathematical Society, Providence, RI, 2008. MR 2009d:11062 Zbl 1165.11042

[Garibaldi and Hoffmann 2006] S. Garibaldi and D. W. Hoffmann, "Totaro's question on zero-cycles on $G_{2}, F_{4}$ and $E_{6}$ torsors", J. London Math. Soc. (2) 73:2 (2006), 325-338. MR 2007g:11049 Zbl 1092.11021

[Gille and Szamuely 2006] P. Gille and T. Szamuely, Central simple algebras and Galois cohomology, Cambridge Studies in Advanced Mathematics 101, Cambridge University Press, Cambridge, 2006. MR 2007k:16033 Zbl 1137.12001

[Knus et al. 1998] M.-A. Knus, A. S. Merkurjev, M. Rost, and J.-P. Tignol, The book of involutions, American Mathematical Society Colloquium Publications 44, American Mathematical Society, Providence, RI, 1998. MR 2000a:16031 Zbl 0955.16001

[Lam 1980] T. Y. Lam, The algebraic theory of quadratic forms, Benjamin/Cummings, Reading, MA, 1980. MR 83d:10022 Zbl 0437.10006

[Merkurjev 1995] A. S. Merkurjev, “Норменный принцип для алгебраических групп”, Algebra i Analiz 7:2 (1995), 77-105. Translated as "The norm principle for algebraic groups", St. Petersburg Mathematical Journal 7:2 (1996), 243-264. MR 96k:20088 Zbl 0859.20039

[Sansuc 1981] J.-J. Sansuc, "Groupe de Brauer et arithmétique des groupes algébriques linéaires sur un corps de nombres”, J. Reine Angew. Math. 327 (1981), 12-80. MR 83d:12010 Zbl 0468.14007 
[Serre 1995] J.-P. Serre, Cohomologie Galoisienne: progrès et problèmes, pp. 229-257, Astérisque 227, Société Mathématique de France, Paris, 1995, Available at http://eudml.org/doc/110186. Séminaire Bourbaki, Vol. 1993/94, Exp. No. 783:4. MR 97d:11063 Zbl 0837.12003

[Springer 1952] T. A. Springer, "Sur les formes quadratiques d'indice zéro", C. R. Acad. Sci. Paris 234 (1952), 1517-1519. MR 13,815j Zbl 0046.24303

[Tits 1992] J. Tits, "Sur les degrés des extensions de corps déployant les groupes algébriques simples", C. R. Acad. Sci. Paris Sér. I Math. 315:11 (1992), 1131-1138. MR 93m:20059 Zbl 0823.20042

[Totaro 2004] B. Totaro, "Splitting fields for E $E_{8}$-torsors", Duke Math. J. 121:3 (2004), 425-455. MR 2005h:11081 Zbl 1048.11031

Received November 28, 2012. Revised February 19, 2013.

JODI BLACK

DEPARTMENT OF MATHEMATICS

BUCKNELL UNIVERSITY

LEWISBURG, PA 17837

UNITED STATES

jodi.black@bucknell.edu

RAMAN PARIMALA

Department of Mathematics ANd Computer SCIENCE

EMORY UNIVERSITY

400 DOWMAN DRIVE W401

ATLANTA, GA 30322

UNITED STATES

parimala@mathcs.emory.edu 


\title{
PACIFIC JOURNAL OF MATHEMATICS
}

\author{
msp.org/pjm
}

Founded in 1951 by E. F. Beckenbach (1906-1982) and F. Wolf (1904-1989)

\section{EDITORS}

Don Blasius (Managing Editor)

Department of Mathematics

University of California

Los Angeles, CA 90095-1555

blasius@math.ucla.edu

\author{
Paul Balmer \\ Department of Mathematics \\ University of California \\ Los Angeles, CA 90095-1555 \\ balmer@math.ucla.edu \\ Robert Finn \\ Department of Mathematics \\ Stanford University \\ Stanford, CA 94305-2125 \\ finn@math.stanford.edu \\ Sorin Popa \\ Department of Mathematics \\ University of California \\ Los Angeles, CA 90095-1555 \\ popa@math.ucla.edu
}

\author{
Vyjayanthi Chari \\ Department of Mathematics \\ University of California \\ Riverside, CA 92521-0135 \\ chari@math.ucr.edu \\ Kefeng Liu \\ Department of Mathematics \\ University of California \\ Los Angeles, CA 90095-1555 \\ liu@math.ucla.edu \\ Jie Qing \\ Department of Mathematics \\ University of California \\ Santa Cruz, CA 95064 \\ qing@ cats.ucsc.edu
}

\section{PRODUCTION}

Silvio Levy, Scientific Editor, production@msp.org

\section{SUPPORTING INSTITUTIONS}

ACADEMIA SINICA, TAIPEI

CALIFORNIA INST. OF TECHNOLOGY

INST. DE MATEMÁTICA PURA E APLICADA

KEIO UNIVERSITY

MATH. SCIENCES RESEARCH INSTITUTE

NEW MEXICO STATE UNIV.

OREGON STATE UNIV.

\author{
STANFORD UNIVERSITY \\ UNIV. OF BRITISH COLUMBIA \\ UNIV. OF CALIFORNIA, BERKELEY \\ UNIV. OF CALIFORNIA, DAVIS \\ UNIV. OF CALIFORNIA, LOS ANGELES \\ UNIV. OF CALIFORNIA, RIVERSIDE \\ UNIV. OF CALIFORNIA, SAN DIEGO \\ UNIV. OF CALIF., SANTA BARBARA
}

\author{
Daryl Cooper \\ Department of Mathematics \\ University of California \\ Santa Barbara, CA 93106-3080 \\ cooper@math.ucsb.edu \\ Jiang-Hua Lu \\ Department of Mathematics \\ The University of Hong Kong \\ Pokfulam Rd., Hong Kong \\ jhlu@maths.hku.hk \\ Paul Yang \\ Department of Mathematics \\ Princeton University \\ Princeton NJ 08544-1000 \\ yang@math.princeton.edu
}

These supporting institutions contribute to the cost of publication of this Journal, but they are not owners or publishers and have no responsibility for its contents or policies.

See inside back cover or msp.org/pjm for submission instructions.

The subscription price for 2014 is US $\$ 410 /$ year for the electronic version, and \$535/year for print and electronic.

Subscriptions, requests for back issues and changes of subscribers address should be sent to Pacific Journal of Mathematics, P.O. Box 4163, Berkeley, CA 94704-0163, U.S.A. The Pacific Journal of Mathematics is indexed by Mathematical Reviews, Zentralblatt MATH, PASCAL CNRS Index, Referativnyi Zhurnal, Current Mathematical Publications and Web of Knowledge (Science Citation Index).

The Pacific Journal of Mathematics (ISSN 0030-8730) at the University of California, c/o Department of Mathematics, 798 Evans Hall \#3840, Berkeley, CA 94720-3840, is published twelve times a year. Periodical rate postage paid at Berkeley, CA 94704, and additional mailing offices. POSTMASTER: send address changes to Pacific Journal of Mathematics, P.O. Box 4163, Berkeley, CA 94704-0163.

PJM peer review and production are managed by EditFLOW ${ }^{\circledR}$ from Mathematical Sciences Publishers.

\section{PUBLISHED BY}

\section{mathematical sciences publishers \\ nonprofit scientific publishing}

http://msp.org/

(C) 2014 Mathematical Sciences Publishers 


\section{PACIFIC JOURNAL OF MATHEMATICS}

Volume $269 \quad$ No. $2 \quad$ June 2014

Totaro's question for simply connected groups of low rank

257

JODI BLACK and RAMAN PARIMALA

Uniform hyperbolicity of the curve graphs

269

BRIAN H. BOWDITCH

Constant Gaussian curvature surfaces in the 3-sphere via loop groups

281

DAVID BRANDER, JUN-ICHI INOGUCHI and SHIMPEI KOBAYASHI

On embeddings into compactly generated groups

305

PIERRE-EMmanuel CAPRACE and Yves CORNULIER

Variational representations for $N$-cyclically monotone vector fields

ALFRED GALICHON and NASSIF GHOUSSOUB

Restricted successive minima

341

MaRTIN HenK and CARSTEN THIEL

Radial solutions of non-Archimedean pseudodifferential equations

355

ANATOLY N. KochubeI

A Jantzen sum formula for restricted Verma modules over affine Kac-Moody

algebras at the critical level

JOHANNES KÜBEL

Notes on the extension of the mean curvature flow

YAN LENG, ENTAO ZHAO and HaORAN ZHAO

Hypersurfaces with prescribed angle function

Henrique F. DE Lima, ERAldo A. Lima JR. and Ulisses L. Parente

Existence of nonparametric solutions for a capillary problem in warped products

JoRGE H. LiRA and GABRIELA A. WANDERLEY

A counterexample to the simple loop conjecture for $\operatorname{PSL}(2, \mathbb{R})$

425

KATHRYN MANN

Twisted Alexander polynomials of 2-bridge knots for parabolic representations

TAKAYUKI MORIFUJI and ANH T. TRAN

Schwarzian differential equations associated to Shimura curves of genus zero

FANG-TING TU

Polynomial invariants of Weyl groups for Kac-Moody groups 